Supporting Information for

\title{
Liquid-like Solids Support Cells in 3D
}

Tapomoy Bhattacharjee ${ }^{1}$, Carmen J. Gil ${ }^{2}$, Samantha L. Marshall ${ }^{1}$, Juan M. Urueña ${ }^{1}$, Christopher S. O'B'ryan', Matt Carstens', Benjamin Keselowsky', Glyn D. Palmer ${ }^{4}$, Steve Ghivizzani ${ }^{4}$, C. Parker Gibbs ${ }^{4}$, W. Gregory Sawyer ${ }^{1,5}$, Thomas E. Angelini ${ }^{1,3 *}$

${ }^{1}$ Department of Mechanical \& Aerospace Engineering, 571 Gale Lemerand Drive, University of Florida, Gainesville FL, 32611

${ }^{2}$ Department of Chemical Engineering, University of Florida, 1030 Center Drive, Gainesville FL, 32611

${ }^{3}$ J. Crayton Pruitt Family Department of Biomedical Engineering, 1275 Center Drive, University of Florida, Gainesville FL, 32611

${ }^{4}$ Department of Orthopaedics and Rehabilitation, University of Florida, 3450 Hull Road, Gainesville FL, 32611

${ }^{5}$ Department of Material Science \& Engineering, University of Florida, Gainesville FL, 32611

Keywords: 3D printing; bioprinting; biowriting; three dimensional cell culture; high throughput screening; tumor engineering; cancer

Number of pages: 6

Number of figures: 8 


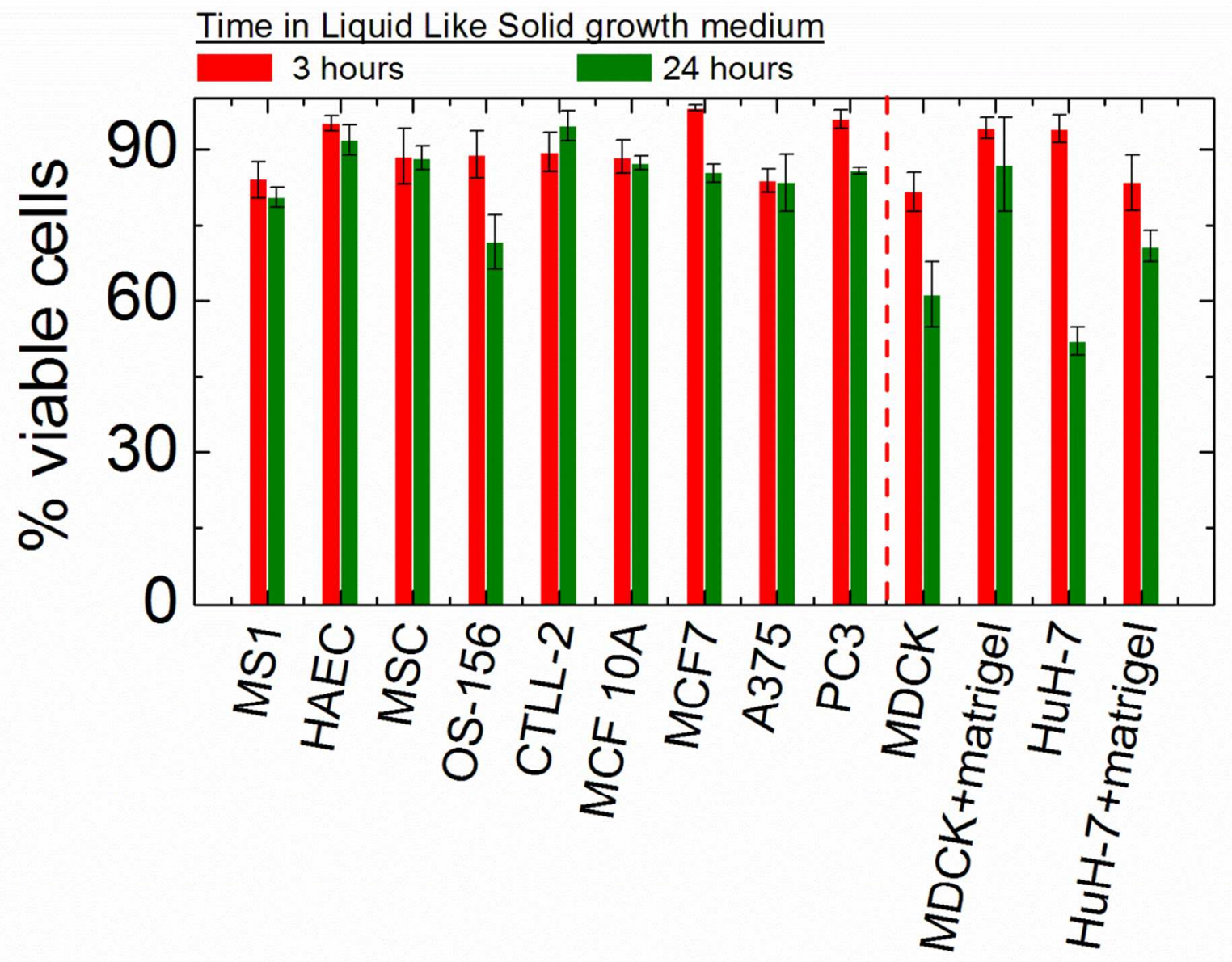

Figure S1. Cell Viability. Eleven different cell lines are dispersed and incubated in liquid like solid medium for 3 hours and 24 hours respectively to explore short term cell viability. ReadyProbes Cell Viability Imaging Kit (Life Technologies) is used to stain all the cells and dead cells. Cell counts (total \& dead) obtained from confocal microscope images are used to determine the viability. Relative to cells harvested from the 2D culture dish ( 3 hour time-point), we have found an average viability of about $94 \%$ after 24 hours (Table 1 in main text). Endothelial cells, MS1 (MILE SVEN 1) and HAEC (Human Aortic Endothelial Cells) along with Mesenchymal Stem Cells (MSC), cytotoxic T cells (CTLL-2) and non-tumorigenic human epithelial cell line MCF10A exhibit an absolute viability of approximately $90 \%$ after incubation inside the LLS medium for 24 hours. Primary osteosarcoma cells (OS) along with other cancer cell lines such as human malignant melanoma (A375), Human Breast Adenocarcinoma (MCF7), Caucasian prostate adenocarcinoma (PC3) also show high viability in LLS medium. Interestingly, to achieve this high viability with Madin Darby Canine Kidney (MDCK) cells and human hepatoma cells $(\mathrm{HuH}-7)$, a supplemental matrix (matrigel) must be co-dispersed with the cells into the LLS medium. 


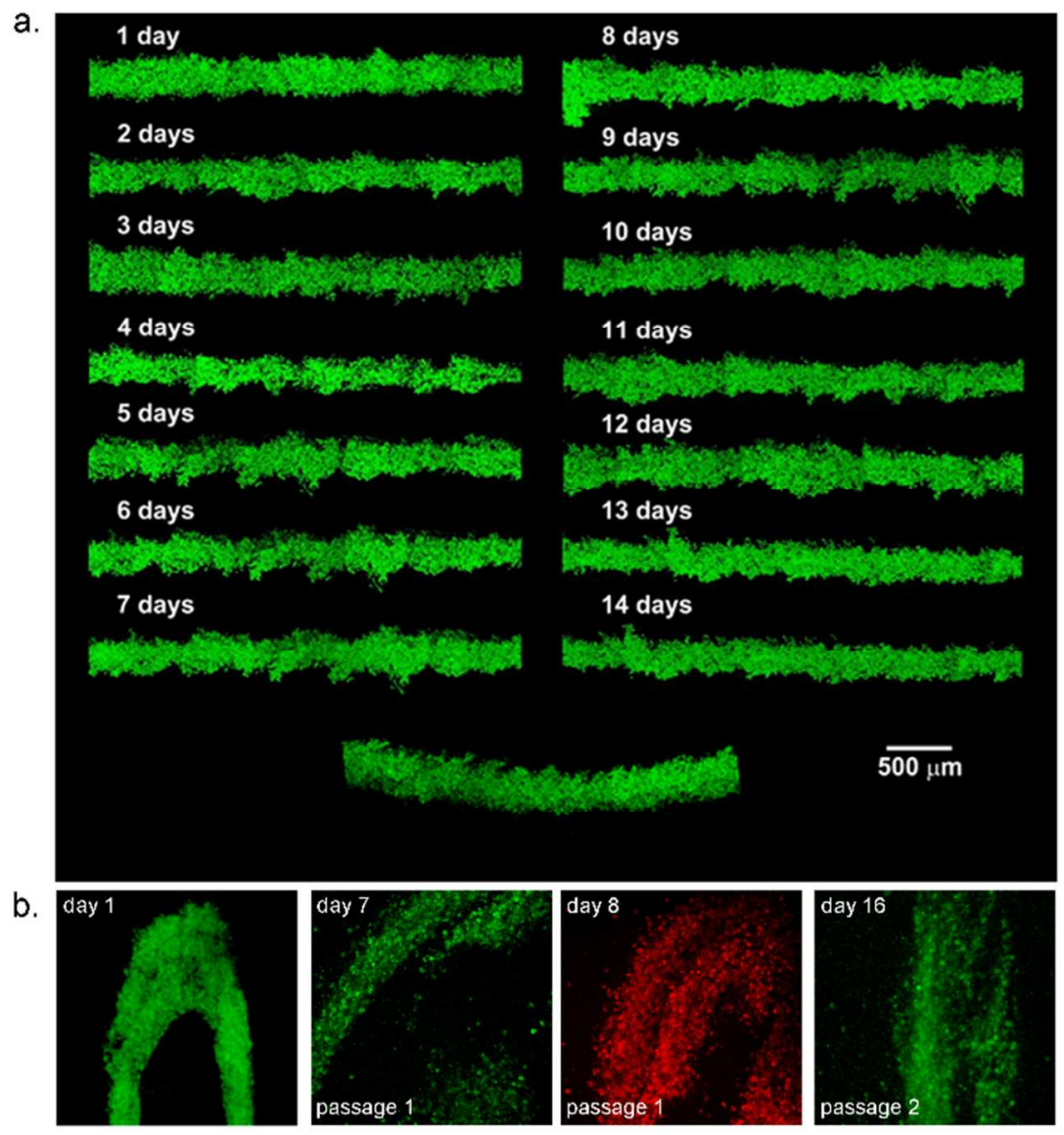

Figure S2. Passaging cells in LLS medium. (a) Cylindrical structures of HuH-7 cells were printed in liquid like solid growth media. This structure was monitored for 2 weeks before removing and imaging in liquid culture media. (b) Aggregates of MCF10A were created inside LLS growth media. The solid structure of MCF10A was dyed with calcein-AM (day 1) and incubated inside the LLS for 7 days. On day 7, this structure was removed and placed inside a fresh LLS bath (day 7, passage 1). This structure was dyed with calcein red on day 8 to confirm the continued metabolic activity of cells and observe the presence of new cells. On day 16, the structure was passaged and dyed with calcein green for a second time, where the persistence of cell viability was observed. 

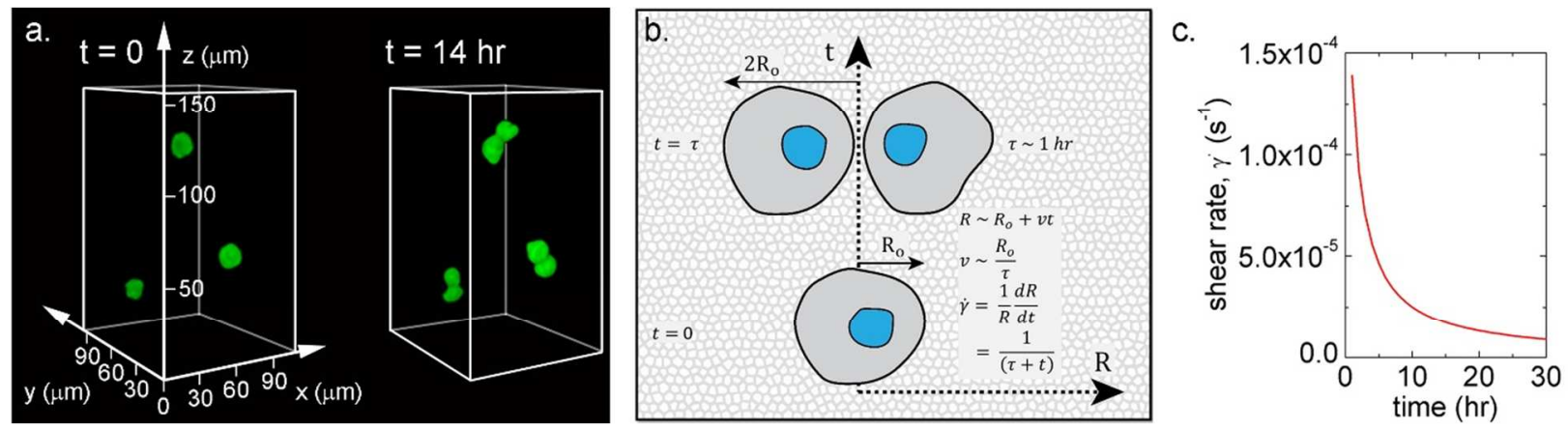

Figure S3. Yield stress and cell division. MCF10A cells can divide while supported by the liquid like solid medium (a). During division as the two daughter cells separate, they strain the surrounding medium by expanding from an initial size-scale, $R_{0}$ to a larger size-scale which we approximate to be $2 R_{0}$. Treating this expansion to scale linearly with time, we estimate a strainrate which depends on the time, $\tau$, over which the daughter cells move apart (b). The maximum shear that occurs during this process is extremely low, falling in a regime where viscous stresses are negligible compared to the Herschel-Bulkley yield stress (c). Thus, isolated cells only have to generate approximately $10 \mathrm{~Pa}$ of stress to divide.

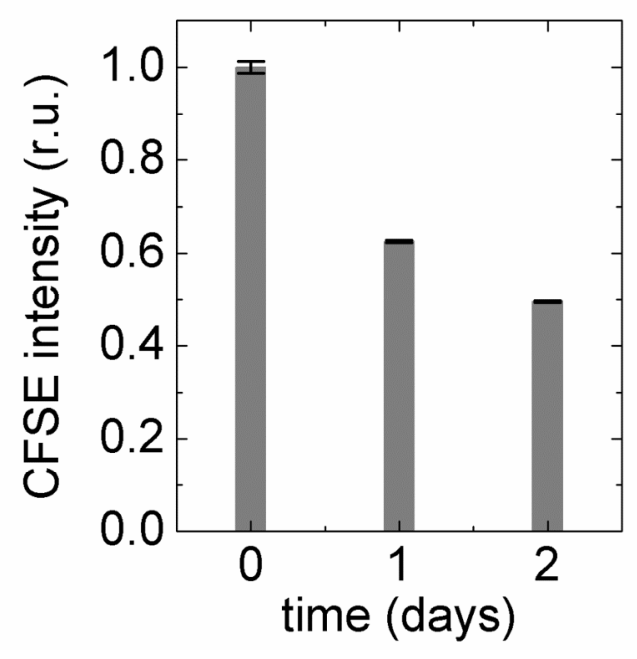

Figure S4. Proliferation. MCF10A cells were dyed with $5 \mu \mathrm{M}$ Carboxy-fluorescein succinimidyl ester (CFSE) and spheroids are printed inside a bath of liquid like solid. Spheroids at three different time points are harvested from the liquid like solid media and dispersed. To measure proliferation, fluorescence intensities of $10^{4}$ cells from these spheroids are measured in a flow cytometer. The fractional decrease in fluorescence intensity is the reciprocal of the number of division cycles that have occurred. Thus, this proliferation study shows a cell doubling time of two days in liquid like solid medium. 


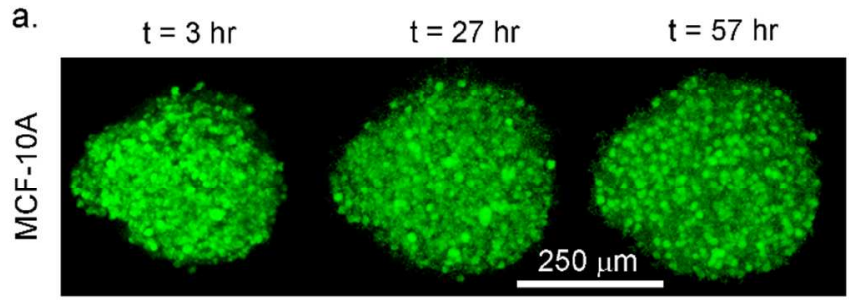

d.

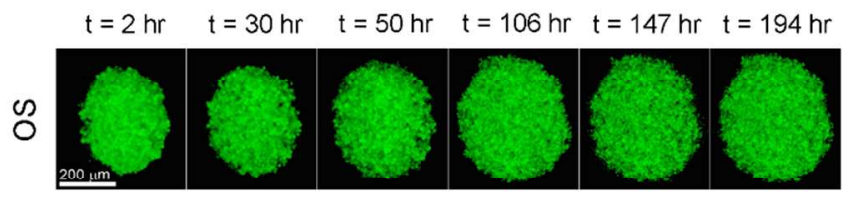

b.

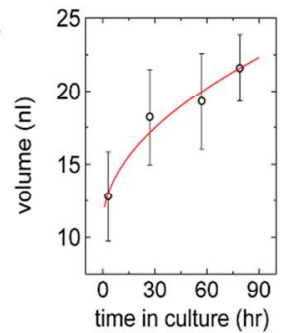

e.

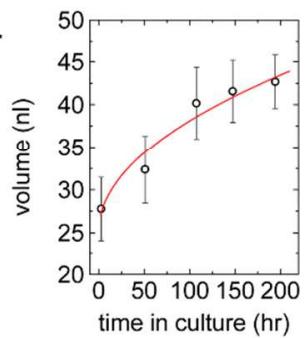

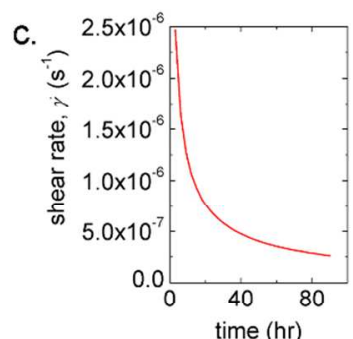

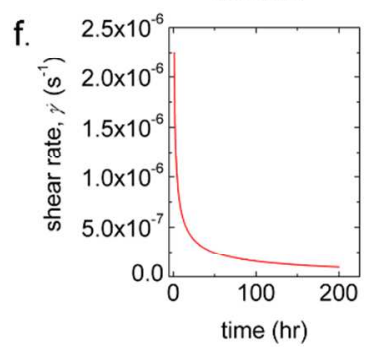

Figure S5. Spheroid growth in liquid like solid medium. MCF10A and primary osteosarcoma spheroids are created in liquid like solid medium and dyed with caldein-AM (a,d). Maximum intensity projections of confocal images are analyzed to measure the spheroid diameters and to determine spheroid volume. Spheroid volume scales approximately like the square-root of time (b,e). Shear rates of these sphere growths are calculated as $\dot{\gamma}=R^{-1} d R / d t$, where $R$ is the spheroid radius and $t$ is time (c,f). The shear rate associated with spheroid growth always falls in the plateau region of the LLS yielding curve which ensures that the spheroid is under a constant stress equal to the yield stress.

a.

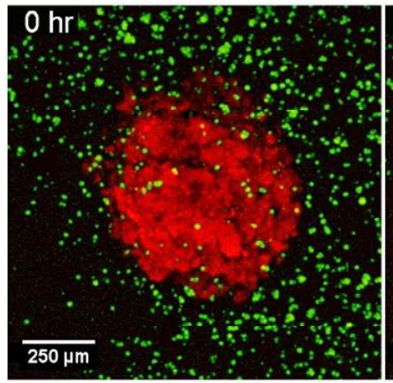

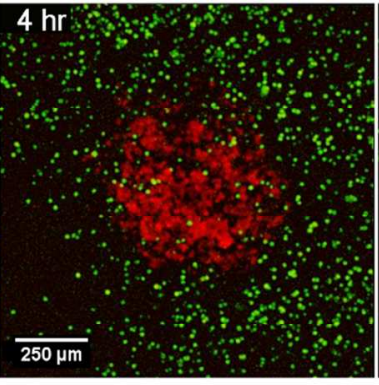

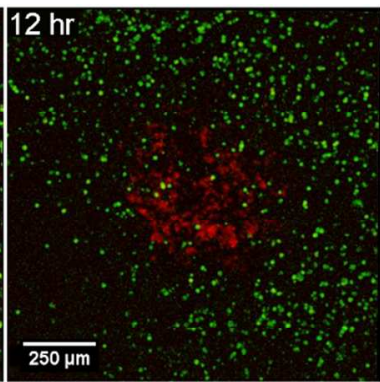

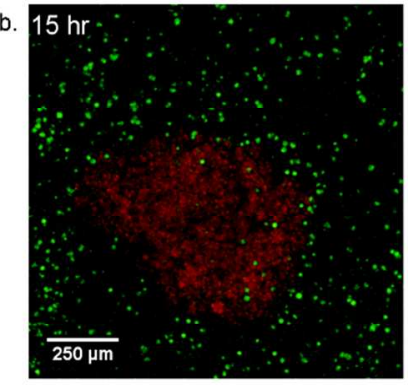

Figure S6. Co-culture of cytotoxic T-cells and 3D printed osteosarcoma tumor. (a) An osteosarcoma tumor (red) is printed in a dispersion of killer T-cells (green). The maximum intensity projection image of the system shows a shrinking tumor with decreasing fluorescence intensity from the vital dye (calcein-red). By contrast, the identical tumors are always observed to grow in the absence of the killer T-cells (ED fig. 7). (b) To check for potential artifacts from photobleaching, we imaged a second tumor that was never previously exposed to light after printing, finding again the reduced red fluorescence, confirming the death of OS cells. 


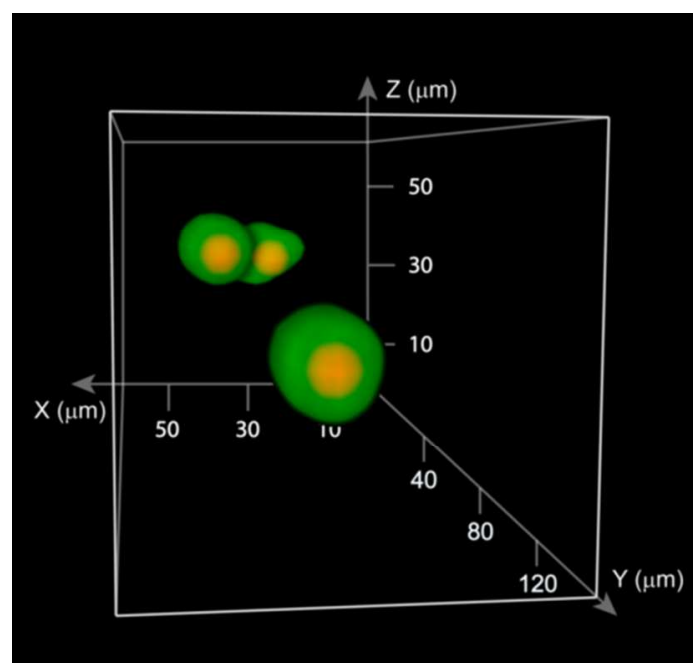

Figure S7. Three dimensional cell tracking. MCF10A cells are dyed with chloromethylfluorescein diacetate (CMFDA) and dispersed in the liquid like solid medium. A time-lapse series of 3D fluorescence images of cells are collected throughout space on an environmentally controlled confocal microscope for 20 hours (green spheres). 3D centroids of individual cells are identified and tracked over all time frames (red centers).

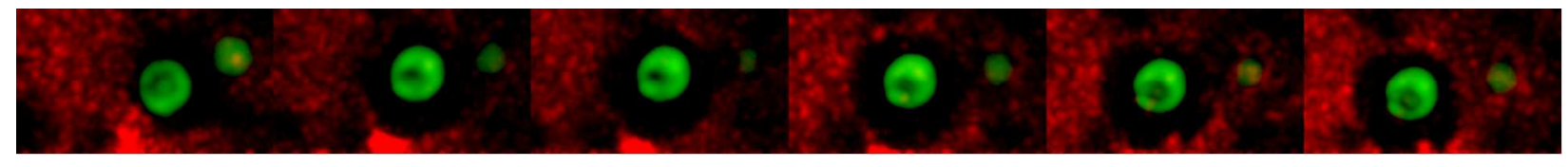

Figure S8. Pericellular matrix in 3D. MCF10A cells are dyed with CMFDA and dispersed into the LLS medium containing red fluorescent polystyrene microspheres $1 \mu \mathrm{m}$ in diameter. The fluorescent microspheres appear to be excluded from a large zone surrounding each cell, which may be a pericellular matrix. Maximum intensity projection images show that the thickness of this excluded region is approximately one cell diameter. 